PAPER

\title{
Fatigue in multiple sclerosis: an example of cytokine mediated sickness behaviour?
}

\author{
C Heesen, L Nawrath, C Reich, N Baver, K-H Schulz, S M Gold
}

See Editorial Commentary, p 2

J Neurol Neurosurg Psychiatry 2006;77:34-39. doi: 10.1136/jnnp.2005.065805

See end of article for authors' affiliations

.....................

Correspondence to: Dr Christoph Heesen, Department of Neurology, University Hospital Eppendorf, Martinistrasse 52, D-20246 Hamburg, Germany; heesen@uke. uni-hamburg.de

Received 17 February 2005 Revised version received 28 May 2005

Accepted 26 June 2005
Background: Fatigue is a major complaint of multiple sclerosis (MS) patients. However, little is known about its pathophysiological mechanisms. Evidence from chronic fatigue syndrome and studies on sickness behaviour suggest that immune and neuroendocrine factors may play a causative role in the development of fatigue.

Methods: We compared whole blood stimulatory capacity for pro- (TNF $\alpha$, IFN $\gamma$ ) and anti-inflammatory cytokines (IL-10) as well as hypothalamo-pituitary-adrenal (HPA) axis function in 15 MS patients with marked fatigue and 15 patients without fatigue as determined by the Fatigue Severity Scale (FSS).

Results: Proinflammatory cytokines were significantly higher (TNF $\alpha$ : $478.9 \vee 228.2 \mathrm{pg} / \mathrm{ml}, \mathrm{p}=0.01$; IFN $\gamma$ : $57.6 \vee 27.8 \mathrm{pg} / \mathrm{ml} ; \mathrm{p}=0.01$ ) in MS patients with fatigue. Furthermore, TNF $\alpha$ values significantly correlated with daytime sleepiness as measured by the Epworth Sleepiness Scale $(r=0.64, p=0.001)$. Controlling for disease activity (as measured by the Cambridge Multiple Sclerosis Basic Score), disease duration, Expanded Disability Status Scale, and depression further increased the correlation of cytokine production and fatigue. HPA axis activity was not related to fatigue but was modestly correlated with cognitive impairment.

Conclusion: Our data suggest that fatigue in MS is at least partially mediated through activation of proinflammatory cytokines. In line with earlier findings, HPA axis dysfunction seems not to be relevant in MS fatigue pathogenesis but appears to be linked to cognitive impairment. Our findings suggest that increased levels of inflammatory cytokines may be involved in MS fatigue. Investigation of cytokine profiles may increase the understanding of fatigue pathogenesis in MS.
M ultiple sclerosis (MS) is an inflammatory and degenerative disease with a presumed autoimmune aetiology. ${ }^{1}$ Fatigue is common in patients with MS. It is also one of the most disabling symptoms, with up to two thirds of patients describing fatigue as their main complaint (for review see Schwid $e t a l^{2}$ ).

Despite the high clinical relevance, there are few studies on the pathophysiology of MS fatigue and the mechanisms are not yet clear. Based on the finding that many proinflammatory cytokines have been shown to induce fatigue and somnolence when administered exogenously, an immune mediated process for symptoms of fatigue has been postulated. ${ }^{3}$ In fact, patients suffering from conditions associated with fatigue, such as chronic fatigue syndrome, ${ }^{4}$ sleep apnea, ${ }^{5}$ glucocorticoid withdrawal syndrome, ${ }^{4}$ and depression, ${ }^{6}$ are known to have altered levels of cytokines in the peripheral circulation, generally skewed towards a $\mathrm{T}$ helper type $\mathrm{l}$ proinflammatory profile.

However, few studies have investigated the inflammatory markers in MS patients with fatigue. Giovannoni et al could not find any correlations between fatigue scores and urinary neopterin, CRP, and sICAM- 1 in a sample of 38 MS patients. However, proinflammatory cytokines such as IFN $\gamma$ and $\mathrm{TNF} \alpha$, which are considered to play a pivotal role in the development of sickness behaviour, ${ }^{6}$ have not been systematically examined in MS in this context.

While fatigue is not clearly associated with disease subtype, duration, or degree of disability in MS, it is linked to depressive symptomatology. ${ }^{8}$ Vice versa, fatigue is a crucial feature of major depression. In recent years it has become clear that the hypothalamo-pituitary adrenal (HPA) axis system is altered in mood disorders, anxiety, and stress associated pathologies. ${ }^{9}$ An altered HPA axis function has also been described in chronic fatigue syndrome, characterised by blunted responses to pharmacological challenge. ${ }^{10}$ A centrally disturbed feedback regulation indicating HPA hyperactivity has been demonstrated in approximately 30\% of MS patients by means of the dexamethasone-CRH (Dex$\mathrm{CRH})$ test. $^{11-13}$ However, a small subgroup with HPA hypoactivity has also been identified. ${ }^{11}$ HPA reactivity in MS patients with prominent fatigue has not yet been studied. Based on evidence from studies in chronic fatigue and depression, a neuroendocrine mechanism of fatigue in MS seems possible. It has been shown that inflammatory cytokines induce activation of the HPA axis. Thus, production of inflammatory mediators within the CNS in MS may not only lead to sickness behaviour and fatigue but also to activation of the HPA axis.

The aim of the present study was to compare proinflammatory (IFN $\gamma, \mathrm{TNF} \alpha$ ) and anti-inflammatory (IL-10) cytokine production as well as HPA axis function in MS patients with and without fatigue. We hypothesised that fatigue would be associated with proinflammatory cytokine production and increased negative feedback of the HPA axis with inadequately low responses to acute stimuli.

\footnotetext{
Abbreviations: ACTH, adrenocorticotropic hormone; AUC, area under the curve; CAMBS, Cambridge Multiple Sclerosis Basic Score; CRH, corticotropin releasing hormone; Dex-CRH test, dexamethasone-CRH test; EDSS, Expanded Disability Status Scale; ESS, Epworth Sleepiness Scale; FSS, Fatigue Severity Scale; HADS, Hospital Anxiety and Depression Scale; HPA axis, hypothalamo-pituitary-adrenal axis; MFIS, modified Fatigue Impact Scale; MS, multiple sclerosis; PHA, phytohaemagglutinin; PPMS, primary progressive MS; RRMS, relapsing remitting MS; SD, standard deviation; SDMT, Symbol Digit Modalities Test; SPMS, secondary progressive MS
} 


\section{METHODS \\ Subjects}

Patients were recruited from our MS database and divided into two groups according to the Fatigue Severity Scale (FSS). ${ }^{14}$ Patients with FSS mean scores $\geqslant 5$ were allocated to the "Fatigue" group, while those with FSS scores $<4$ were allocated to the "No fatigue" group. We recruited only patients with definitive MS according to Poser criteria, ${ }^{15}$ and without steroid therapy within the past 4 weeks. Patients with clinically evident psychiatric disease were excluded. The study was approved by the local ethics committee. All subjects were told about the purpose of the study and written informed consent was obtained prior to entering the study.

\section{Clinical scores and questionnaires}

All patients underwent neurological screening to classify disease course according to Lublin et al.$^{16}$ Expanded Disability Status Scale (EDSS) scores ${ }^{17}$ were rated by an experienced neurologist in our outpatient clinic. Cognitive impairment was evaluated using the Symbol Digit Modalities Test (SDMT). ${ }^{18}$ The number of correct answers was compared to that of an age and education adjusted healthy control cohort, and individual scores were then transformed into standard deviations (SD) below or above those normal values. Disease impact was furthermore classified according to the Cambridge Multiple Sclerosis Basic Score $\left(\right.$ CAMBS) ${ }^{19}$ for the dimensions disability (graded 0-5: 0, fully independent to 5, totally dependent), relapse (graded 0-5: 0, stable to 5, relapse which requires hospitalisation), progression in the last 12 months (graded 0-5: 0, stable to 5, marked malignant progression), and handicap (graded 0-5: 0, no effect on role in life to 5, incapable of any useful role). Affective symptomatology was assessed with the German version of the Hospital Anxiety and Depression Scale (HADS). ${ }^{20}$ We further assessed fatigue with the modified Fatigue Impact Scale (MFIS). ${ }^{21}$ The Epworth Sleepiness Scale (ESS) ${ }^{22}$ was used to assess daytime sleepiness.

\section{Whole blood cytokine stimulation}

Cytokines (IFN $\gamma, \mathrm{TNF} \alpha, \mathrm{IL}-10$ ) were determined in a whole blood short term culture. Briefly $400 \mu \mathrm{l}$ of whole blood was added to $3.2 \mathrm{ml}$ of RPMI (endotoxin content $<0.01 \mathrm{EU} / \mathrm{ml}$ ) supplemented with glutamate and streptomycin/penicillin $(100 \mu \mathrm{g} / \mathrm{ml})$ in sterile $5 \mathrm{ml}$ tubes. We used $10 \mu \mathrm{g} / \mathrm{ml}$ phytohaemagglutinin (PHA) as a stimulant for IFN $\gamma$ and $\mathrm{TNF} \alpha$, and $25 \mu \mathrm{g} / \mathrm{ml}$ PHA as a stimulant for IL-10. Control cultures without stimulation were also prepared. Tubes were capped and incubated at $37^{\circ} \mathrm{C}$ for $24 \mathrm{~h}$. Optimal stimulation conditions had been determined previously using different amounts of PHA $(2.5-25 \mu \mathrm{g} / \mathrm{ml})$ and incubation times (3$24 \mathrm{~h}$ ). Mean \pm SD delta changes comparing unstimulated and stimulated cultures were $108 \pm 53.2$ for IL-10, $42.3 \pm 41.5$ for IFN $\gamma$, and $330.6 \pm 229.1$ for $\mathrm{TNF} \alpha$, which were in the ranges of previous studies. The caps were removed and samples centrifuged. Supernatants were collected and frozen at $-80^{\circ} \mathrm{C}$ until ELISA was performed. All tests were performed according to the manufacturer (human IL-10 ELISA, human TNF-alpha ELISA Version 2, and human IFN-gamma ELISA; Bender Med Systems, Vienna, Austria). Probes were analysed in duplicates. The plate was read on the E-max ELISA processor at $450 \mathrm{~nm}$ with $620 \mathrm{~nm}$ blank filter. The reported sensitivity of the IL-10 ELISA was $2 \mathrm{pg} / \mathrm{ml}$ with an intra- and interassay variation of $5 \%$ and $6 \%$. The IFN $\gamma$ assay had a sensitivity of $1.5 \mathrm{pg} / \mathrm{ml}$ and an intra- and interassay variation of $4.5 \%$ and $5.7 \%$, respectively. The reported sensitivity of the TNF $\alpha$ ELISA was $5.8 \mathrm{pg} / \mathrm{ml}$ with an intra- and interassay variation of 6.9 and $7.4 \%$, respectively.

\section{Dex-CRH test}

Dex-CRH tests were performed as described earlier. ${ }^{11}$ Briefly, patients were pretreated with $1.5 \mathrm{mg}$ oral dexamethasone at 23:00 h the night before the test. An i.v. cannula was inserted at 14:30 h and kept patent. Blood was taken at 14:30, 15:00, 15:30, 16:00, and 16:30 h. At 15:00 h, $100 \mu \mathrm{g}$ synthetic corticotropin releasing hormone $(\mathrm{CRH}$; Ferring, Kiel, Germany) reconstituted in $1 \mathrm{ml} 0.09 \%$ saline was injected as an i.v. bolus. The five blood samples were drawn in prechilled tubes, anticoagulated with EDTA, and immediately centrifuged at $4^{\circ} \mathrm{C}$. Plasma was frozen and stored at $-20^{\circ} \mathrm{C}$.

\section{Hormone assays}

An immunoluminometric two step assay (Nichols Advantage, San Juan Capistrano, CA) was used for the determination of adrenocorticotropic hormone (ACTH) in plasma. Two monoclonal antibodies, one luminescent labelled and the other immobilised on the inner surface of the tube, recognise different binding sites on corticotropin to form a sandwichtype complex bound to the tube. The luminescence signal is directly proportional to the corticotropin concentration. The detection limit was $1 \mathrm{pg} / \mathrm{ml}$. The intra- and interassay coefficients of variation were $2.7 \%$ and $6.4 \%$, respectively. Cortisol was measured by a dual antibody chemiluminescence assay using the Elecsys System 2010 (Roche, GrenzachWhylen, Germany) kit. The detection limit was $3.6 \mathrm{ng} / \mathrm{ml}$. Intra- and interassay coefficients of variation were $1.3 \%$ and $1.5 \%$, respectively.

\section{Statistical analysis}

Baseline cortisol and ACTH as well as the area under the curve (AUC) over time by trapezoidal estimation were used from the Dex-CRH test. Because cytokine and endocrine parameters were not normally distributed, we used log transformed values in all analyses.

Group differences (fatigue and no fatigue according to FSS cut offs) in demographic as well as disease describing factors, cytokines, and endocrine parameters were analysed using parametric $t$ tests for independent samples. The $\chi^{2}$ test was used for nominal variables.

For further differentiation of the association of MS fatigue and neuroendocrine and cytokine parameters, we analysed Pearson correlation coefficients with an MS specific measure of fatigue (MFIS) and a score of daytime sleepiness (ESS).

In order to control for potential confounding factors such as disease severity and affective symptomatology, we also computed partial correlations statistically removing the effect of disability (EDSS, CAMBS), interferon medication (interferon $v$ other or no treatment), and disease duration as well as depressive symptoms (HADS). The possible effect of the disease course was investigated by computing correlations for patients with relapsing remitting MS and patients with chronic disease (secondary progressive MS and primary progressive MS) separately.

Results are given as means $\pm S D$. All analyses were conducted with statistical software (SPSS 11.0; SPSS, Chicago, IL). A p value less than 0.05 was considered significant. A p value less than 0.10 was accepted in order to detect trends.

\section{RESULTS}

\section{Demographic data}

Thirty patients were included in the study (table 1). Patients in the two groups differed strongly according to their score on the FSS. Patients with fatigue also had significantly higher mean values in the fatigue scale (MFIS) and in the daytime sleepiness scale (ESS). In addition to the fatigue scores, there were other significant differences between the two groups. The fatigue group showed significantly higher levels on the 
EDSS, and higher scores on the CAMBS subscales disability, progression, and handicap. The fatigue group further showed more depressive symptoms on the HADS. Four patients had scores above the cut off for overt depression (that is, a score of 12 or higher on the HADS depression subscale). Nineteen patients were receiving immunomodulatory treatment.

In the fatigue group, there was a statistical trend towards longer disease duration $(p=0.09)$. Disease courses also differed: in the fatigue group, six patients had relapsing remitting MS (RRMS), eight secondary progressive MS (SPMS), and one primary progressive MS (PPMS), whereas in the no fatigue group, 11 patients had RRMS, two SPMS, and two PPMS $\left(\chi^{2}=5.40, p=0.07\right)$. Five patients in the fatigue group were treated with interferons compared to six patients in the group without fatigue $\left(\chi^{2}=0.144, p=0.71\right)$.

\section{Cytokine findings}

MS patients with fatigue based on the FSS grouping had significantly higher mean IFN $\gamma$ and TNF $\alpha$ production capacity than patients without fatigue (IFN $\gamma$ : $57.6 \pm 41.6 v$ $27.8 \pm 37.1 \mathrm{pg} / \mathrm{ml}, \quad \mathrm{p}=0.01 ; \quad \mathrm{TNF} \alpha: 478.9 \pm 209.7 \quad v$ $228.2 \pm 208.1 \mathrm{pg} / \mathrm{ml}, \mathrm{p}=0.01)$. IL-10 production showed no significant difference between the two groups $(128.2 \pm 47.0 \mathrm{~V}$ $97.0 \pm 52.3 \mathrm{pg} / \mathrm{ml}, \mathrm{p}=0.10$ ) (fig $\mathrm{l}$ ).

MFIS scores correlated significantly with IFN $\gamma$ and TNF $\alpha$ but not with IL-10 production (IFN $\gamma$ : $\mathrm{r}=0.45, \mathrm{p}=0.02$; TNF $\alpha: \quad r=0.45, \quad p=0.03 ; \quad$ IL-10: $r=0.26, \quad p=0.20 ;$ see fig $2 \mathrm{~A}, \mathrm{~B})$.

The total score and the cognitive and physical subscales of the MFIS correlated equally with the IFN $\gamma$ and TNF $\alpha$ findings (data not shown). TNF $\alpha$ was the only cytokine correlating significantly with daytime sleepiness as measured by the ESS (TNF $\alpha$ : $r=0.55, p=0.01$, see fig $2 C$; IFN $\gamma: r=0.33, p=0.09$; IL-10: $\mathrm{r}=0.24, \mathrm{p}=0.24$ ).

It is important to note that the correlations observed between fatigue and cytokine levels were not due to group differences in disease severity, duration, or depressive symptoms. When statistically controlling for EDSS, disease duration (years), disease severity (CAMBS-Handicap, CAMBS-Disability) and progression (CAMBS-Progression), depressive symptoms (HADS), and interferon treatment, the partial correlation coefficients actually increased (IFN $\gamma$ :

Table 1 Demographic data of the study sample

\begin{tabular}{llll}
\hline & Fatigue & No fatigue & $\mathbf{p}$ \\
\hline $\mathrm{n}$ & 15 & 15 & \\
Age & $46.6 \pm 11.69$ & $42.87 \pm 10.17$ & 0.36 \\
Gender, M/F & $6 / 9$ & $6 / 9$ & $>0.99^{*}$ \\
Years with MS & $13.80 \pm 9.68$ & $8.60 \pm 5.57$ & 0.09 \\
EDSS & $4.36 \pm 1.49$ & $2.30 \pm 1.84$ & 0.002 \\
FSS & $6.08 \pm 0.68$ & $1.51 \pm 0.69$ & $<0.001$ \\
MFIS & $58.07 \pm 10.55$ & $7.53 \pm 8.08$ & $<0.001$ \\
ESS & $10.53 \pm 4.71$ & $3.27 \pm 2.37$ & $<0.001$ \\
HADS & $7.93 \pm 3.30$ & $3.13 \pm 3.50$ & 0.001 \\
CAMBS & & & \\
Disability & $2.93 \pm 0.25$ & $1.93 \pm 0.79$ & $<0.001$ \\
Relapse & $1.33 \pm 0.48$ & $1.60 \pm 0.63$ & 0.21 \\
Progression & $2.40 \pm 0.91$ & $1.53 \pm 0.63$ & 0.01 \\
$\quad$ Handicap & $3.60 \pm 0.82$ & $2.00 \pm 1.06$ & $<0.001$ \\
SDMT & $-0.40 \pm 1.72$ & $0.13 \pm 1.30$ & 0.35 \\
Disease course & 6 RR, 8 SP, 1 PP & 11 RR, 2 SP, 2 PP & $0.07^{*}$ \\
Treatment & 6 immune & 9 immune & $0.51^{*}$ \\
& modulators, & modulators & \\
& 4 immuno- & & \\
& suppressants & & \\
\hline
\end{tabular}

Data are given as means $\pm S D ; p$ values according to $t$ test or $\chi^{2}$ test* . CAMBS, Cambridge Multiple Sclerosis Basic Score; EDSS, Expanded Disability Status Scale; ESS, Epworth Sleepiness Scale; FSS, Fatigue Severity Scale; MFIS, modified Fatigue Impact Scale; HADS, Hospital Anxiety and Depression Scale; SDMT, Symbol Digit Modalities Scale. $\mathrm{r}=0.68, \mathrm{p}=0.001 ; \mathrm{TNF} \alpha: \mathrm{r}=0.54, \mathrm{p}=0.03)$. When correlations were computed for RRMS and chronic MS (SPMS and PPMS) separately, the coefficients actually increased (RRMS: IFN $\gamma: \mathrm{r}=0.57, \mathrm{p}=0.03$; TNF $\alpha$ : $\mathrm{r}=0.54, \mathrm{p}=0.06$; SPMS and PPMS: IFN $\gamma: \mathrm{r}=0.47, \mathrm{p}=0.15$; TNF $\alpha: \mathrm{r}=0.53, \mathrm{p}=0.12$ ).

\section{Endocrine findings}

In the whole group, only three patients showed nonsuppression in the Dex-CRH tests according to the cortisol cut off of $40 \mathrm{pg} / \mathrm{ml}^{23}$ Patients with fatigue and without fatigue showed similar response patterns of baseline ACTH or cortisol as well as AUC ACTH or cortisol in the Dex-CRH test (fig 3). After controlling for disability, disease duration, depression, disease course, and interferon medication, only the correlation between cortisol baseline and fatigue as measured by the FSS showed a trend $(r=0.38, p=0.08)$.

Neither baseline ACTH or cortisol nor AUC ACTH or cortisol correlated significantly with cytokine levels (all coefficients below $\mathrm{r}=0.29$ after controlling for the confounding factors mentioned above). Further analysis of Dex-CRH tests in our sample disclosed six patients with low cortisol $(<10 \mathrm{pg} / \mathrm{ml})$ after dexamethasone pretreatment and nearly no stimulatory effect of ACTH. While these patients had greater cognitive impairment, they did not have higher fatigue scores, disease activity, or disability in general.

\section{Effects of depression and cognitive impairment}

HADS scores did not correlate with any of the measured cytokines (TNF $\alpha$ : $r=0.08, p=0.70$; IFN $\gamma$ : $r=0.08, p=0.67$; IL-10: $r=-0.001, p=0.99$ ) or endocrine parameters (ACTH baseline: $\mathrm{r}=-0.10, \mathrm{p}=0.59 ;$ AUC: $\mathrm{r}=-0.01, \mathrm{p}=0.95$; cortisol baseline: $r=-0.07, p=71$; AUC: $r=0.03, p=0.84$ ).

However, cognitive impairment was significantly correlated with suppression of ACTH after dexamethasone pretreatment (ACTH baseline: $r=-0.43, p=0.02$ ) but not with cortisol at baseline $(r=-0.20, p=0.30)$. Furthermore, cognitive impairment showed a modest correlation with hyperresponsiveness of ACTH AUC in the Dex-CRH test $(\mathrm{r}=-0.32, \mathrm{p}=0.08$; see fig 4$)$, while cortisol AUC was not correlated $(r=-0.09, p=0.63)$. Again, correlation coefficients increased after controlling for confounding factors (ACTH baseline: $\mathrm{r}=-0.53, \mathrm{p}=0.01$; cortisol baseline: $\mathrm{r}=-0.37, \mathrm{p}=0.08$; ACTH AUC: $\mathrm{r}=-0.43, \mathrm{p}=0.04$; cortisol AUC: $r=-0.22, p=0.32$ ).

\section{DISCUSSION}

This study investigated cytokine and endocrine characteristics in MS patients with marked fatigue according to the FSS compared to patients with low FSS scores. IFN $\gamma$ and $\mathrm{TNF} \alpha$ production capacity in response to PHA was significantly enhanced in MS patients with fatigue. IL-10 production seemed less affected. TNF $\alpha$ moderately correlated with disease activity as well as with fatigue. TNF $\alpha$ also correlated with daytime sleepiness as measured by the ESS. We thus hypothesise that $\mathrm{TNF} \alpha$ is a key mediator of MS fatigue. Furthermore, daytime sleepiness might be a clinical indicator for a cytokine mediated fatigue syndrome.

While there were significant differences regarding disease severity between fatigued and non-fatigued patients, it is unlikely that the differences are responsible for the observed effects in cytokine levels. There were more patients with secondary progressive disease in the fatigue group. As a result, average disease duration was significantly longer and mean EDSS was higher. Later disease stages are generally considered to have less inflammatory activity, particularly in the secondary progressive phase. ${ }^{1}$ It would, therefore, be counterintuitive to expect higher levels of proinflammatory cytokines in a group of patients with later-stage disease. In line with this argument, statistically adjusting for measures 


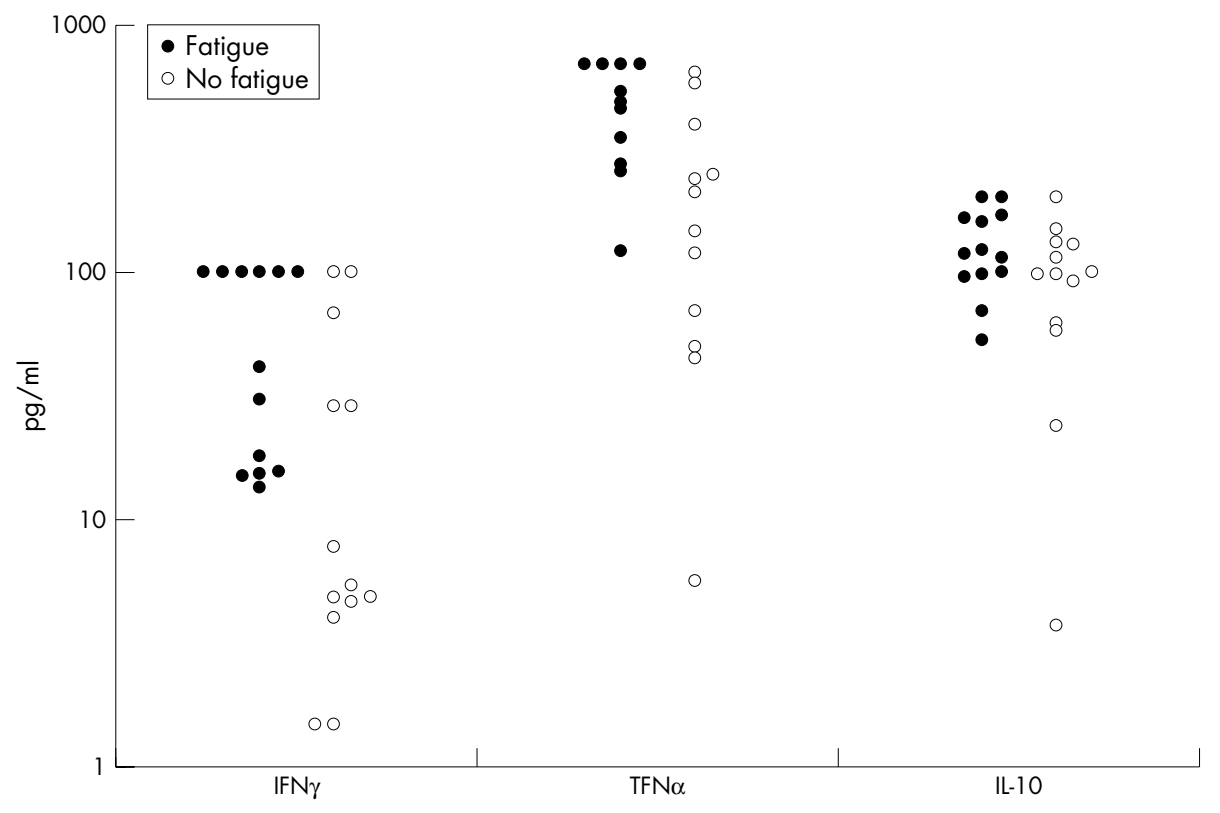

Figure 1 Pro-inflammatory (IFN $\gamma, \mathrm{TNF} \alpha$ ) and anti-inflammatory (IL-10) cytokines in MS patients with and without fatigue. Each measurement is represented by a dot. See Results section for mean values and statistical differences.

of disease severity (EDSS, duration of disease, CAMBSHandicap, CAMBS-Disability) actually further increased the direct correlations of IFN $\gamma$ and TNF $\alpha$ production capacity and fatigue scores. Thus, it appears unlikely that our findings are confounded by disease severity.

Our findings suggest a key role for TNF $\alpha$ in MS fatigue. Anti$\mathrm{TNF} \alpha$ treatment strategies have recently been shown to suppress fatigue very effectively in sleep apnea syndrome, ${ }^{24}$ stressing the relevance of $\mathrm{TNF} \alpha$ in fatigue pathogenesis. Our findings are supported by a recent study by Flachenecker $e t$ al, ${ }^{25}$ which reported fatigue scores were correlated with TNF $\alpha$ mRNA production in $27 \mathrm{MS}$ patients with FSS scores $\geqslant 4$. IFN $\gamma$ or IL-10 levels, however, were not related to fatigue in their study. Cytokine production capacity upon whole blood stimulation represents more of a functional test than a measurement of actual transcribed cytokines, which might render our method more sensitive. These methodological differences might explain the divergent findings concerning IFN $\gamma$.

In a previous study on neuropsychological fatigue in $\mathrm{MS}^{26}$ we found baseline cytokine production levels were not altered in 23 MS patients compared to 25 healthy controls. After a 40 min cognitive stressor, MS patients showed lower
IFN $\gamma$ production capacities, while IL-10 responses were unaltered. This finding was in accordance with our previous experimental stress studies demonstrating attenuated cytokine responses in MS upon acute experimental physical or psychological stress. ${ }^{27} 28$ In the abovementioned study, MS patients with fatigue were compared to healthy controls, thus precluding any conclusion on fatigue mechanisms. Furthermore, fatigue scores were lower than in the present study.

Since MS fatigue was associated with enhanced proinflammatory cytokine production capacity in the present study, anti-inflammatory treatment may represent an alternative treatment option to administering agents which promote wakefulness, such as modafinil, for therapy in MS fatigue. This concept requires further investigation.

Fatigue was not significantly correlated with any parameter of the Dex-CRH test. Thus, HPA axis dysregulation does not appear to be a general mediator of MS fatigue. This finding confirms previous studies which found no correlation of fatigue scores with Dex-CRH test results. ${ }^{12}{ }^{13} 29$ However, all these reports, including the study presented here, had small sample sizes, thus limiting generalisability.
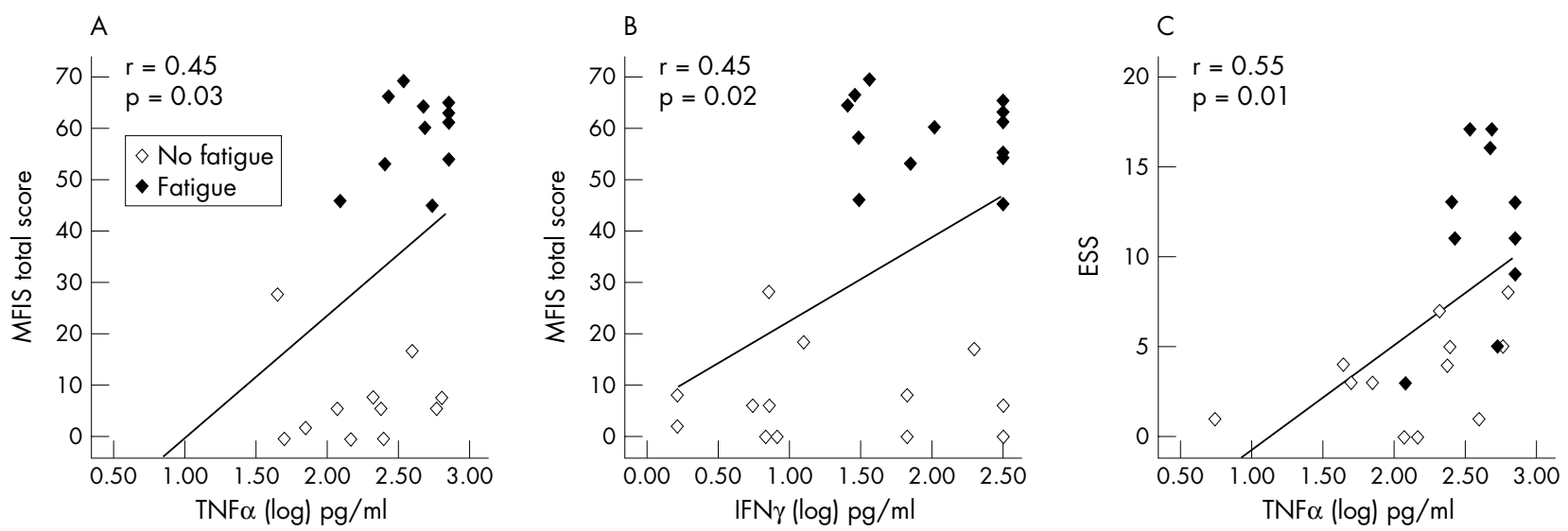

Figure 2 (A, B) Correlation of MFIS (total score) with (A) TNF $\alpha(n=23)$ and (B) IFN $\gamma(n=26)$. (C) Correlation of ESS with TNF $\alpha(n=23)$. 

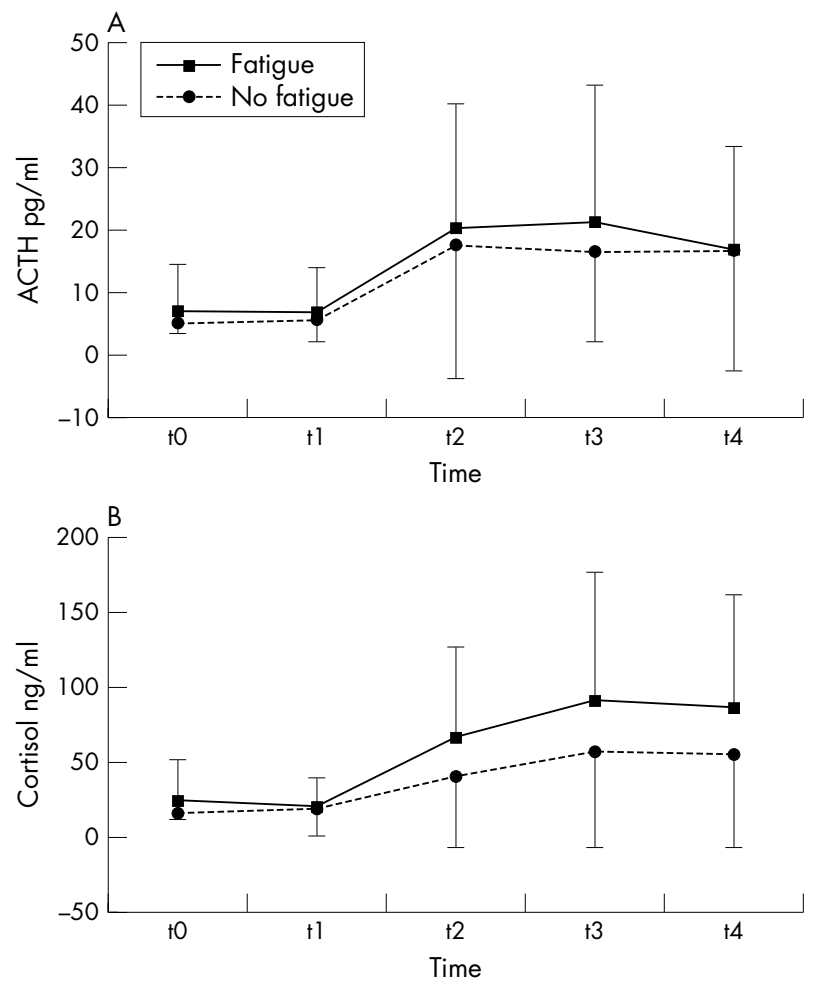

Figure 3 Time course of ACTH and cortisol plasma concentration in the Dex-CRH test in MS patients with $(n=15)$ and without $(n=15)$ fatigue (mean values with SD).

In a recent study by Gottschalk et al, ${ }^{30}$ increased HPA activity was reported in MS patients with fatigue. While Gottschalk et al analysed data from a similar number of patients and used an almost identical cut off value for fatigue, their sample exclusively consisted of RRMS patients, most of whom were untreated and had a shorter disease duration as well as lower overall disability. In contrast, our sample contained a significant portion of chronic progressive patients, and more than $50 \%$ of our subjects had received immunomodulatory or immunosuppressive treatment. This has likely increased the interindividual variation in HPA measures and in turn decreased our statistical power for this part of the analyses. It is also conceivable that fatigue in RRMS is associated with different factors than fatigue in the more progressive phase of the disease. This may in part explain the stronger association of fatigue and HPA axis function in the study by Gottschalk et al ${ }^{30}$ compared to our data. Clearly, larger studies of homogeneous patient groups are necessary to clarify the contribution of inflammation, neurodegeneration, neuropsychiatric symptoms, and HPA activity to fatigue in MS.

In the present study, we were able to replicate our previous finding of an association of cognitive dysfunction with hyperreactivity in the Dex-CRH test. ${ }^{12}$ Further studies comparing cognitively impaired and unimpaired MS patients using the Dex-CRH test and MRI are under the way to clarify the mechanisms of this association. As depression scores were low in our sample with only four patients above the clinical cut off score of 12, no conclusion concerning the relation between the HPA axis and depressive symptomatology in MS can be drawn from this study.

So far, attempts to establish objective markers of MS fatigue have been unsuccessful. Recruitment of patients to study fatigue has to rely on patient self report, which mixes symptoms and compensation strategies. By definition, there

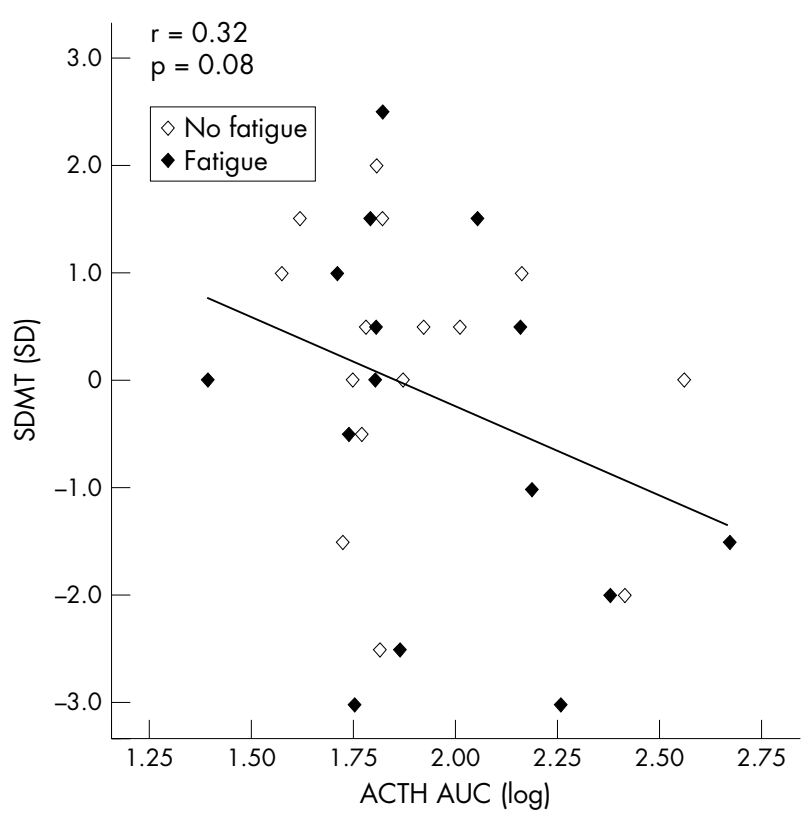

Figure 4 Scatter plot of ACTH AUC (log) corresponding to SDMT (with $\mathrm{SD})$. ACTH AUC values were log transformed and are given in arbitrary units (trapezoidal estimation).

is considerable overlap of fatigue and depressive symptoms and self report measures may be heavily affected by recall bias. $^{2}$ The FSS is one of the most commonly used screening scales for fatigue and has been shown to have acceptable reliability and validity in $\mathrm{MS}^{31}$ However, it is a short screening tool that is biased towards assessing physical fatigue. $^{31}$

We conclude that higher proinflammatory cytokine production capacity may in part be responsible for symptoms of fatigue in MS patients. Our data suggest that TNF $\alpha$ in particular may be important for daytime sleepiness. Fatigue significantly interferes with activities of daily living and is described by patients as one of the most disabling symptoms. ${ }^{2}$ In addition to tests of central motor excitability, inhibition pathways, and sympathetic function, cytokine production capacity could be useful to classify patients. Potentially, the investigation of cytokine profiles may increase understanding of fatigue pathogenesis in MS and ultimately help to develop better symptomatic treatment.

\section{ACKNOWLEDGEMENTS}

We thank W Tessmer for excellent help in performing the cytokine studies and R Jung, Department of Clinical Chemistry for analysis of cortisol and ACTH.

\section{Authors' affiliations}

C Heesen, L Nawrath, N Bauer, S M Gold, Department of Neurology, University Hospital Eppendorf, Hamburg, Germany

C Reich, K-H Schulz, Institute of Medical Psychology and Transplantation Center, University Hospital Eppendorf, Martinistrasse 52, 20246 Hamburg, Germany

S M Gold, Multiple Sclerosis Center, Department of Neurology and Cousins Center for Psychoneuroimmunology, Neuropsychiatric Institute, UCLA School of Medicine, 710 Westwood Plaza, Los Angeles, CA 90095, USA

This study was supported by the Gemeinnützige Hertie-Stiftung (grant nos: 1.319.120-01-01 and 1.01.1/03/013). Stefan M Gold is in part supported by a grant from the Deutsche Forschungsgemeinschaft (DFG; grant no: GO 1357/1-1)

Competing interests: none declared 


\section{REFERENCES}

1 Lucchinetti C, Brueck W, Parisi J, et al. Heterogeneity of multiple sclerosis lesions: implications for the pathogenesis of demyelination. Ann Neurol 2000:47:707-17

2 Schwid SR, Covington M, Segal BM, et al. Fatigue in multiple sclerosis: current understanding and future directions. J Rehabil Res Dev 2002;39:21 1-24.

3 Konsman JP, Parnet P, Dantzer R. Cytokine-induced sickness behaviour: mechanisms and implications. Trends Neurosci 2002;25:154-9.

4 Papanicolau DA, Amsterdam JD, Levine S, et al. Neuroendocrine aspects of chronic fatigue syndrome. Neuroimmunomodulation 2004;11:65-74

5 Vgontzas AN, Papanicolaou DA, Bixler EO, et al. Sleep apnea and daytime sleepiness and fatigue: relation to visceral obesity, insulin resistance, and hypercyłokinemia. J Endocrinol Metab 2000;85:1151-8.

6 Lee BN, Dantzer R, Langley KE, et al. A cytokine-based neuroimmunologic mechanism of cancer related symptoms. Neuroimmunomodulation 2004;11:279-92.

7 Giovannoni G, Thompson AJ, Miller DH, et al. Fatigue is not associated with raised inflammatory markers in multiple sclerosis. Neurology 2001;58:1134.

8 Kroencke DC, Lynch SG, Denney DR. Fatigue in multiple sclerosis: relationship to depression, disability, and disease pattern. Mult Scler 2000;6(2):131-6.

9 Gold PW, Gabry KE, Yasuda MR, et al. Divergent endocrine abnormalities in melancholic and atypical depression: clinical and pathophysiologic implications. Endocrinol Metab Clin North Am 2002;31(1):37-62.

10 Cleare AJ. The HPA axis and the genesis of chronic fatigue syndrome. Trends Endocrinol Metab 2003;15:55-9.

11 Grasser A, Möller A, Backmund A, et al. Heterogeneity of hypothalamicpituitary-adrenal system response to a combined dexamethasone-CRH test in multiple sclerosis. Exp Clin Endocrinol 1996;104:31-7.

12 Heesen C, Gold SM, Raji A, et al. Cognitive impairment correlates with hypothalamo-pituitary-adrenal axis dysregulation in multiple sclerosis. Psychoneuroendocrinology 2002;27:505-17

13 Fassbender K, Schmidt R, Mossner R, et al. Mood disorders and dysfunction of the hypothalamic-pituitary-adrenal axis in multiple sclerosis: association with cerebral inflammation. Arch Neurol 1998;55:66-72.

14 Krupp LB, LaRocca NG, Muir-Nash J, et al. The fatigue severity scale. Application to patients with multiple sclerosis and systemic lupus erythematosus. Arch Neurol 1989;46:1121-3.

15 Poser CM. New diagnostic criteria for multiple sclerosis: guidelines for research protocols. Ann Neurol 1983;13:227-31.

16 Lublin FD, Reingold SC for the National Multiple Sclerosis Society (USA) Advisory Committee on Clinical Trials of New Agents in Multiple Sclerosis.
Defining the clinical course of multiple sclerosis: results of an international survey. Neurology 1996;46:907-11.

17 Kurtzke JM. Rating neurological impairment in multiple sclerosis: an expanded disability status scale. Neurology 1983;33:1444-52.

18 Smith A. The Symbol Digit Modalities Test. Learn Disord 1968;3:83-91.

19 Mumford C, Compston A. Problems with rating scales for multiple sclerosis: a novel approach - the CAMBS score. J Neurol 1993;240:209-15.

20 Zigmond AS, Snaith RP. The Hospital Anxiety and Depression Scale. Acta Psychiatr Scand 1983;67:361-70.

21 Multiple Sclerosis Council for Clinical Practice Guidelines. Fatigue and multiple sclerosis: evidence-based management strategies for fatigue in multiple sclerosis. Washington, DC: Paralyzed Veterans of America, October, 1998.

22 Hoddes E, Zarcone V, Smythe H, et al. Quantification of sleepiness: a new approach. Psychophysiology 1973;10:431-6.

23 Von Bardeleben U, Holsboer F. Cortisol response to a continued dexamethasone human corticotrophin-releasing hormone challenge in patients with depression. J Neuroendocrinol 1989;1:485-8.

24 Vgontzas AN, Zoumakis E, Lin HM, et al. Marked decrease in sleepiness in patients with sleep apnea by etanercept, a tumor necrosis factor- $\alpha$ antagonist. $J$ Endocrinol Metab 2004;89:4409-13.

25 Flachenecker P, Bihler I, Weber F, et al. Cytokine mRNA expression in patients with multiple sclerosis and fatigue. Mult Scler 2004;10:165-9.

26 Heesen C, Koehler G, Gross R, et al. Altered cytokine responses to cognitive stress in multiple sclerosis patients with fatigue. Mult Scler 2005;1 1:51-7.

27 Heesen C, Schulz H, Schmidt M, et al. Endocrine and cytokine responses to acute psychological stress in multiple sclerosis. Brain Behav Immun 2002;16:282-7.

28 Heesen C, Gold SM, Hartmann S, et al. Endocrine and cytokine response to standardized physical stress in patients with multiple sclerosis and healthy controls. Brain Behav Immun 2003;17:473-81.

29 Then Bergh F, Kümpfel T, Trenkwalder C, et al. Dysregulation of the hypothalamo-pituitary-adrenal axis is related to the clinical course of MS. Neurology 1999;53:772-7.

30 Gottschalk M, Kümpfel T, Flachenecker $P$, et al. Fatigue and regulation of the hypothalamo-pituitary-adrenal axis in multiple sclerosis. Arch Neurol 2005;62:277-80

31 Tellez N, Rio J, Tintore M, et al. Does the Modified Fatigue Impact Scale offer a more comprehensive assessment of fatigue in MS? Mult Scler 2005; 11:198-202. 